\title{
Atom-Centered Potentials to Describe Dispersion Forces in Density Functional Theory
}

\author{
I-Chun Lin§ and Ursula Röthlisberger ${ }^{*}$ \\ §SCS Mettler-Toledo Award Winner (Oral Presentation)
}

\begin{abstract}
London dispersion forces are of primordial importance in chemical and biological systems. The KohnSham formalism of density functional theory combined with many popular approximated exchange-correlation functionals either provides sporadic results or fails completely to account for these forces. Dispersion-corrected atom-centered potentials mimic the effect of these forces via atomic orbital-dependent nonlocal potentials whose parameters are obtained by calibrating against references of chosen accuracy. This scheme has shown encouraging outcomes despite its empiricism; the aim of this communication is to give a brief review on the development and applications to date of this promising approach.
\end{abstract}

Keywords: Density functional theory · Dispersion forces · Dispersion-corrected atom-centered potentials

London dispersion forces, also known as dispersion forces or van der Waals (vdW) forces, arise from the transient dipoles all molecules possess as a result of the fluctuations in the instantaneous positions of electrons. They act between separated atoms/molecules even in the absence of charges or permanent electric moments; they play a crucial role in colloidal systems, in noble-gas chemistry and in soft matter generally, contributing significantly to phenomena such as solvation, physisorption, molecular recognition, as well as the stability and conformational variability of molecular crystal and biomacromolecules.

${ }^{*}$ Correspondence: Prof. U. Röthlisberger

Laboratory of Computational Chemistry and

Biochemistry

Ecole Polytechnique Fédérale de Lausanne (EPFL)

$\mathrm{CH}-1015$ Lausanne

Tel.: +41216930321

Fax: +41216930320

E-mail: ursula.roethlisberger@epfl.ch
Without taking dispersion forces into account, proteins may be predicted to be unstable and relative energies of various polypeptide conformations might be largely in error. ${ }^{[1]}$ Even for systems in which stronger intermolecular forces (e.g. direct electrostatic interactions) seem to dominate, these very weak forces may still be pivotal; for example, the relative energies of two phases in ionic materials are often sufficiently sensitive to the dispersion interactions to have a substantial effect on the transition pressure. ${ }^{[2]}$

The Kohn-Sham formulation of density functional theory (DFT) ${ }^{[3,4]}$ has proved itself as one of the most popular and versatile methods for condensed phase studies owing to its reasonable accuracy and affordable computational cost. DFT, in principle, is exact, if the true expression for the exchangecorrelation functionals were known. Yet with many exchange-correlation functionals in practical use such as the local density approximation or generalized gradient approximations, DFT either provides sporadic results or fails completely to account for dispersion forces. ${ }^{[5-10]}$ Most functionals are based on the local electron density, its local gradient and sometimes even the local kinetic energy density. As dispersion interactions contribute to the interaction energy even at distances where electron overlap is negligible, these functionals fail by construction to reproduce these interactions and are unable to describe correctly the leading $r^{6}$ dispersion interaction term due to spontaneous dipolar interactions.

In consequence, if dispersion forces are of any importance in the system of interest, they must be recovered in the calculations to grant the results any credibility. New algorithms for efficient calculations of these weak forces have been the focus of many recent works. ${ }^{[11-17]}$ From the practical point of view, any proposed scheme that is to be generally applicable to a wide range of chemically and biologically interesting systems needs to be system independent (transferable) and computationally tractable. Here, we concentrate on one recently introduced approach - dispersion-corrected atom-centered potentials (DCACPs) ${ }^{[18]}$ - which represent the effect of dispersion forces via atomic orbital-dependent potentials whose two adjustable parameters are obtained by calibrating against references of desired accuracy. DCACPs provide a promising approach to include these forces in a fully self-consistent manner within current DFT frameworks.

\section{A Brief Outline of the DCACP Concept}

In this approach, a penalty functional $P$ composed of two energy- and one nuclear-force-dependent terms is introduced ${ }^{[19]}$ 
(Eqn. (1)) where $r$ min and $r$ mid are the intermolecular equilibrium and midpoint (where the interaction energy is half of the equilibrium energy) distances of the reference calculations. $E^{\text {ref }}$ is the reference interaction energy, and $\omega_{I}$ is a weighting factor chosen such that contributions from the energy and the force terms $\left(\mathrm{F}_{I}\right)$ for atom $I$ have the same order of magnitude. Only forces along the intermolecular interaction axis are considered.

$$
\begin{aligned}
\min _{\left\{\sigma_{i}\right\}} \mathcal{P}(r)= & \min _{\left\{\sigma_{i}\right\}}\left[\left|E^{\mathrm{ref}}\left(r_{\min }\right)-E\left(r_{\min },\left\{\sigma_{i}\right\}\right)\right|^{2}+\right. \\
& \left|E^{\mathrm{ref}}\left(r_{\text {mid }}\right)-E\left(r_{\text {mid }},\left\{\sigma_{i}\right\}\right)\right|^{2}+ \\
& \left.\sum_{I} w_{I}\left|\mathrm{~F}_{I}\left(r_{\min },\left\{\sigma_{i}\right\}\right)\right|^{2}\right]
\end{aligned}
$$

This penalty functional is minimized by variationally tuning the parameters $\left\{\sigma_{1}\right\}$ that define the atom-centered nonlocal potentials at $\left\{\mathbf{R}_{I}\right\}$ with the normalized projector $p_{l}(r)$ that is proportional to $\left.r^{l} \exp \left(-r^{2} / 2 \sigma_{2}^{2}\right)\right]$ (Eqn. (2)). $r=\left|\mathbf{r}-\mathbf{R}_{I}\right|$ is the distance from the position of nucleus $I . Y_{l m}$ denotes a spherical harmonic.

$$
v_{I}^{\mathrm{DCACP}}\left(\mathbf{r}, \mathbf{r}^{\prime}\right)=\sum_{m=-l}^{+l} Y_{l m}(\hat{\mathbf{r}}) p_{l}(r) \sigma_{1} p_{l}\left(r^{\prime}\right) Y_{l m}^{*}\left(\hat{\mathbf{r}}^{\prime}\right)
$$

It has been found that one projector is sufficient to obtain accurate results for the reference and test systems. To ensure there is no interference with the atomic pseudopotentials centered at $\mathbf{R}_{I}$, any angular momentum component not occupied in the atomic pseudopotentials can, in principle, be used. By tuning $\sigma_{1}$ and $\sigma_{2}, P$ is minimized in such a way that the reference equilibrium interaction energy and distance as well as the midpoint interaction energy are optimally reproduced for a chosen calibration system. The current generation of DCACPs has been standardized to the rarely used component $l$ $=3$ and has been calibrated against $\operatorname{CCSD}(\mathrm{T})$ (with large basis set) or full CI references for small vdW complexes. For a more detailed description, one is referred to ref. ${ }^{[19]}$

\section{The DCACP Library[19]}

A library of DCACPs for rare gas atoms ( $\mathrm{He}, \mathrm{Ne}, \mathrm{Ar}$ and $\mathrm{Kr}$ ) and commonly encountered biological elements (hydrogen, carbon, nitrogen and oxygen) in combination with BLYP, ${ }^{[20,21]} \mathrm{BP}[20,22]$ and $\mathrm{PBE}^{[23]}$ exchangecorrelation functionals has been generated against carefully chosen calibration systems that fulfil the following conditions:

a) the system is small enough so that highlevel reference calculations are tractable,

Table 1. Equilibrium interaction energies $\left(E_{\mathrm{min}}, \mathrm{kcal} / \mathrm{mol}\right)$ and distances $\left(r_{\min }, \AA\right)$ of the rare-gas dimers (results taken from ref. [19]).

\begin{tabular}{|c|c|c|c|c|c|}
\hline & $\begin{array}{l}\left(\mathrm{H}_{2}\right)_{2} \\
E_{\min }\end{array}$ & $r_{\min }$ & $\begin{array}{l}\left(\mathrm{C}_{6} \mathrm{H}_{6}\right)_{2} \\
E_{\min } r_{\text {min }}\end{array}$ & $\begin{array}{l}\left(\mathrm{N}_{2}\right)_{2} \\
E_{\min } \\
r_{\min }\end{array}$ & $\begin{array}{l}\left(\mathrm{CO}_{2}\right)_{2} \\
E_{\min } r_{\text {min }}\end{array}$ \\
\hline DCACP-BLYP & 0.052 & 3.62 & $1.73 \quad 4.0$ & $0.20 \quad 3.9$ & $0.47 \quad 3.4$ \\
\hline DCACP-BP & 0.052 & 3.60 & $1.74 \quad 3.9$ & $0.20 \quad 3.8$ & $0.45 \quad 3.4$ \\
\hline DCACP-PBE & 0.046 & 3.57 & $1.71 \quad 3.9$ & $0.20 \quad 3.8$ & $0.47 \quad 3.4$ \\
\hline ref. $[25,26]$ & 0.045 & 3.49 & $1.70 \quad 3.9$ & $0.20 \quad 3.8$ & $0.46 \quad 3.3$ \\
\hline
\end{tabular}

$\begin{array}{lllllllll} & \mathrm{He}_{2} & & \mathrm{Ne}_{2} & & \mathrm{Ar}_{2} & & \mathrm{Kr}_{2} & \\ & E_{\text {min }} & r_{\text {min }} & E_{\text {min }} & r_{\text {min }} & E_{\text {min }} & r_{\text {min }} & E_{\text {min }} & r_{\text {min }} \\ & 0.020 & 3.0 & 0.094 & 3.0 & 0.247 & 3.8 & 0.322 & 4.2 \\ \text { DCACP-BLYP } & 0.022 & 3.1 & 0.105 & 3.2 & 0.250 & 3.8 & 0.322 & 4.2 \\ \text { DCACP-BP } & 0.021 & 3.0 & 0.077 & 3.2 & 0.248 & 3.8 & 0.317 & 4.2 \\ \text { DCACP-PBE } & 0.020 & 3.0 & 0.082 & 3.2 & 0.249 & 3.8 & 0.322 & 4.2 \\ \text { ref. [24] } & & & & & & & & \end{array}$

Table 2. Equilibrium interaction energies $\left(E_{\min }, \mathrm{kcal} / \mathrm{mol}\right)$ and distances $\left(r_{\min }, \AA\right)$ of $\left(\mathrm{H}_{2}\right)_{2},\left(\mathrm{C}_{6} \mathrm{H}_{6}\right)_{2}$, $\left(\mathrm{N}_{2}\right)_{2}$ and $\left(\mathrm{CO}_{2}\right)_{2}$ (results taken from ref. [19]).

b) the interaction energy is dominated by the balance between Pauli repulsion and dispersion forces, and

c) the electronic structures of the monomers in the complex are well described by the exchange-correlation functional employed. Table 1 and 2 show the results for the chosen calibration systems.

Tests on the monomers of the calibration systems have shown that DCACPs improve the descriptions of intermolecular interactions without introducing any significant distortions on intramolecular geometries and electronic structures. They can also readily be used alongside pseudopotentials other than the originally employed analytic Goedecker-Teter-Hutter type; ${ }^{[27]}$ a numerical version included as an extra channel (f channel in this case) in Troullier-Martins pseudopotentials ${ }^{[28]}$ can be downloaded from $h t t p: / /$ lcbcpc21.epfl.ch/dcacp/dcacp.html.

The values of DCACP parameters depend heavily on the performance of the underlying exchange-correlation functionals. For BP and BLYP in which spurious dispersion interactions are entirely absent, ${ }^{[5,6]}$ DCACPs always provide an attractive correction to the underlying repulsive functional, in line with the idea of a dispersionmotivated correction. As a result, these combinations, in our view, are preferable over the occasional superior performance of DCACP-PBE due to the fact that they offer more clean-cut interpretations (pure PBE provides some spurious interactions for vdW complexes).

\section{Transferability Tests ${ }^{[19,29-32]}$}

The study on a wide range of $\mathrm{vdW}$ complexes of aliphatic hydrocarbons molecules and crystals of aromatic hydrocarbon com- pounds has illustrated the excellent transferability of DCACPs for different hybridization states of carbon, demonstrating that only one DCACP per element is sufficient, irrespective of the hybridization state. ${ }^{[30]}$ Furthermore, the success in predicting the energetics and structural properties of benzene crystal and graphene sheets confirms the applicability of DCACPs in condensed phase. Quantitative agreement with highlevel results can be obtained, as demonstrated by the potential energy surface of benzene dimer in Fig. 1.

When testing against a well-established benchmark set of noncovalently bound dimers of nucleic acids and amino acids, ${ }^{[33]}$ unsigned mean errors of less than $1.6 \mathrm{kcal} /$ mol compared to the best $a b$ initio data available (extrapolated $\operatorname{CCSD}(\mathrm{T})$ complete basis set limit) are obtained. [31] The overall descriptions for systems that are bound predominantly by interactions other than dispersion forces (e.g. water clusters (hydrogen bonding) and formaldehyde dimer (dipole-dipole interactions)) are, in general, further improved upon including DCACPs. ${ }^{[19,32]}$ The results on small vdW clusters, water clusters up to hexamers, and complexes of aromatic biomolecules and amino acids indicate that DCACPs display a strong transferability to systems other than the calibration ones, i.e. once calibrated, the same DCACP can be applied in different chemical environments with comparable accuracy.

\section{Some Applications ${ }^{[34,35]}$}

The DCACP approach has already been applied to a number of biologically or chemically relevant systems. ${ }^{[34-37]}$ Two such examples, aromatic heterocycle (the 


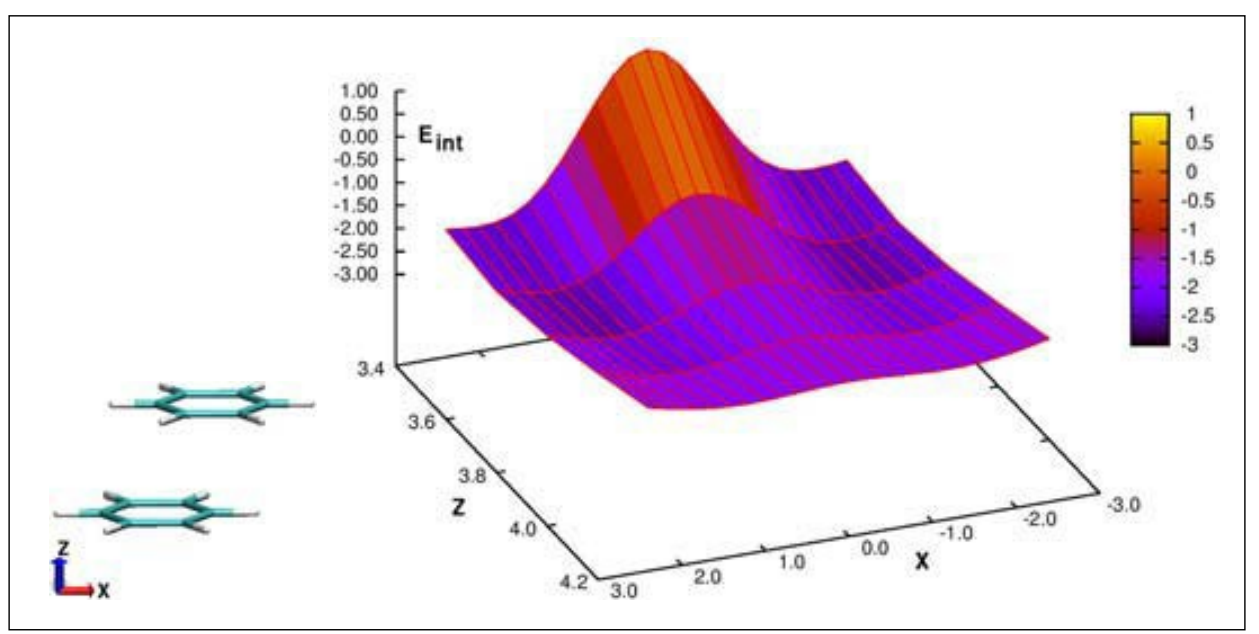

Fig. 1. Interaction energy $\left(E_{\mathrm{int}}, \mathrm{kcal} / \mathrm{mol}\right)$ surface of the parallel-displaced benzene dimer at various $x$ and $z$ distances (both in $\AA$ ) predicted by DCACP-BLYP. The global minimum is at $\left(x, z, E_{\text {int }}\right)=( \pm 1.76$ $3.6,-2.60)$; the estimated $\operatorname{CCSD}(T) /$ aug-cc-pVQZ* result is $( \pm 1.67,3.6,-2.63)^{[26]}$.

anti-cancer agent ellipticine) intercalated in between four nucleobases ${ }^{[34]}$ and the application of DCACPs on liquid water, ${ }^{[35]}$ are represented in Fig. 2.

In the first study, the DCACP-BLYP approach predicts a strongly bound interaction energy profile for the ellipticine intercalation process with a stabilization of nearly $40 \mathrm{kcal} / \mathrm{mol}$ when fully intercalated; whereas estimates based on BLYP only are purely repulsive, showing that a proper description of dispersion forces is an absolute necessity to obtain even a qualitatively correct picture. A preliminary energetic analysis using a slightly smaller model also supports the experimentally observed preference for ellipticine to intercalate in between the GC pairs over the AT pairs. A detailed atomistic investigation of a biological system often requires the knowledge of the electronic structure; one attractive feature of $a b$ initio calculations is thus that one can investigate the effects of complexation on the electronic structure, a phenomenon which is inaccessible to classical force field simulations. The frontier orbitals of the ellipticine-d(CG) ${ }_{2}$ complex show significant changes upon intercalation as compared to the corresponding nonintercalated nucleobases. These changes in electronic structure should have a pronounced influence on the electron/charge transfer properties of DNA, which are of great interest for studies on DNA wire and DNA repair processes, ${ }^{[38,39]}$ to name just a few. The importance of $\mathrm{vdW}$ interactions in liquid water is probed in the second study which shows that DCACPs lead to a distinct improvement in the theoretical description of the structural and dynamical properties of this universal system. ${ }^{[35]}$

Owing to the relative infancy of the DCACP approach, there are still plenty of questions left to be addressed; for example, the performance of DCACPs on chemical reactions, such as the prediction of reaction barriers, remains to be tested. In addition, DCACPs in their current form do not give

the asymptotic $r^{-6}$-tail. Tests on many different systems, however, have shown that the shape of the interaction profile is closely reproduced within distances of up to $5 \AA$. The introduction of the midpoint term in the penalty functional is the first step taken for an improved long-range behavior, but ways to include the correct asymptotic dispersion tails should be investigated. Furthermore, the remarkable success of DCACPs in describing dispersion-dominated systems calls for a more rigorous physical interpretation of this approach based on fundamental principles.

$A b$ initio molecular dynamics simulations have been instrumental in elucidating microscopic mechanisms in an accurate and unbiased manner in various disciplines. The predictive power, however, depends heavily on the accuracy of the chosen functional. The DCACP approach allows one to achieve accuracy similar to the coupled-cluster reference calculations without a large computational overhead. The ability of DCACPs to adapt to the chemical environments is partially due the fact that DCACPs are electronic potentials, and many-body interactions are implicitly included, offering an advantage over the pairwise approach of empirical vdW corrections. The availability of a library for most commonly encountered elements in chemical/biological systems, with a further extension to the library on the way, renders the DCACP approach a practical way to achieve high accuracy without incurring a prohibitive computational penalty. Realistic DFT-based quantum mechanical $/ \mathrm{molec}$ ular mechanical calculations for dispersiondominated systems, such as adsorption on surface, $\pi-\pi$ stacking and hydrophobic effects, can now be envisaged.

\section{Acknowledgements}

Financial support from the Swiss National Science Foundation (Grant No. 200020108063/1) is gratefully acknowledged. We thank $\mathrm{M}$. D. Coutinho-Neto, C. Felsenheimer, A. P. Seitsonen, I. Tavernelli and O. A. von Lilienfeld for discussions.

Received: February 25, 2008

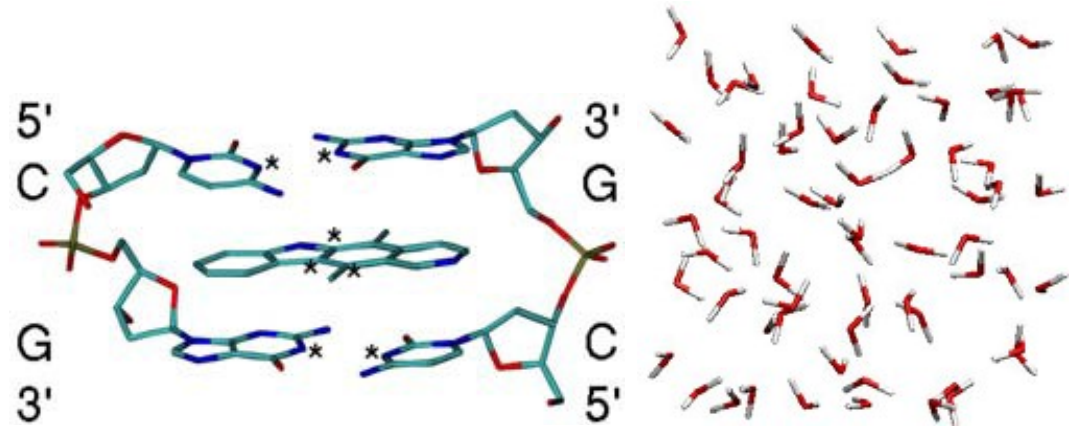

(a) ellipticine-d(CG) $)_{2}$

(b) liquid water

Fig. 2. Graphical representations of the systems investigated

T. Frauenheim, S. Suhai, $J$.

[1] M. Elstner, T. Frauenheim, S. Suhai, J. 29.

[2] P. A. Madden, R. Heaton, A. Aguado, S. Jahn, J. Mol. Struct. (THEOCHEM) 2006, $771,9$.

[3] P. Hohenberg, W. Kohn, Phys. Rev. 1964, 136, B864.

[4] W. Kohn, L. J. Sham, Phys. Rev. 1965, 140, A1133.

[5] S. Kristyán, P. Pulay, Chem. Phys. Lett. 1994, 229, 175.

[6] J. M. Pérez-Jordá, A. D. Becke, Chem. Phys. Lett. 1995, 233, 134.

[7] Y. Zhang, W. Pan, W. Yang, J. Chem. Phys. 1997, 107, 7921. 
[8] D. C. Patton, M. R. Pederson, Int. J. Quantum Chem. 1998, 69, 619.

[9] T. van Mourik, R. J. Gdanitz, J. Chem. Phys. 2002, 116, 9620.

[10] J. Tao, J. P. Perdew, J. Chem. Phys. 2005, $122,114102$.

[11] W. Kohn, Y. Meir, D. E. Makarov, Phys. Rev. Lett. 1998, 80, 4153.

[12] M. Dion, H. Rydberg, E. Schröder, D. C. Langreth, B. I. Lundqvist, Phys. Rev. Lett. 2004, 92, 246401.

[13] S. Grimme, J. Comput. Chem. 2004, 25, 1463.

[14] J. F. Dobson, J. Wang, B. P. Dinte, K. McLennan, H. M. Le, Int. J. Quantum Chem. 2005, 101, 579.

[15] A. J. Misquitta, R. Podeszwa, B. Jeziorski, K. Szalewicz, J. Chem. Phys. 2005, 123, 214103.

[16] A. D. Becke, E. R. Johnson, J. Chem. Phys. 2006, 124, 014104.

[17] Y. Zhao, D. G. Truhlar, J. Chem. Theory Comput. 2007, 3, 289.

[18] O. A. von Lilienfeld, I. Tavernelli, U. Rothlisberger, D. Sebastiani, Phys. Rev. Lett. 2004, 93, 153004.

[19] I-C. Lin, M. C. Coutinho-Neto, C. Felsenheimer, O. A. von Lilienfeld, I. Tavernelli, U. Rothlisberger, Phys. Rev. B 2007, 75, 205131.

[20] A. D. Becke, Phys. Rev. A 1988, 38, 3098.

[21] C. Lee, W. Yang, R. G. Parr, Phys. Rev. B 1988, 37, 785 .

[22] J. P. Perdew, Phys. Rev. B 1986, 33, 8822.

[23] J. P. Perdew, K. Burke, M. Ernzerhof, Phys. Rev. Lett. 1996, 77, 3865.

[24] N. Kurita, H. Sekino, Int. J. Quantum Chem. 2003, 91, 355.
[25] H. Lavendy, J. M. Robbe, and J. P. Flament, Chem. Phys. Lett. 1992, 196, 377.

[26] M. O. Sinnokrot and C. D. Sherrill, J. Phys. Chem. A 2004, 108, 10200.

[27] S. Goedecker, M. Teter, J. Hutter, Phys. Rev. B 1996, 54, 1703.

[28] N. Troullier, J. L. Martins, Phys. Rev. B 1991, 43, 1993.

[29] O. A. von Lilienfeld, I. Tavernelli, U. Rothlisberger, D. Sebastiani, Phys. Rev. $B$ 2005, 71, 195119.

[30] E. Tapavicza, I-C. Lin, O. A. von Lilienfeld, I. Tavernelli, M. D. CoutinhoNeto, U. Rothlisberger, J. Chem. Theory Comput. 2007, 3, 1673.

[31] I-C. Lin, U. Rothlisberger, Phys. Chem. Chem. Phys. 2008, DOI: 10.1039/ b718594d.

[32] I-C. Lin, A. P. Seitsonen, I. Tavernelli, M. D. Coutinho-Neto, U. Rothlisberger, 2008, to be submitted.

[33] P. Jurečka, J. Šponer, J. Černý, P. Hobza, Phys. Chem. Chem. Phys. 2006, 8, 1985.

[34] I-C. Lin, O. A. von Lilienfeld, M. D. Coutinho-Neto, I. Tavernelli, U. Rothlisberger, J. Phys. Chem. B 2007, $111,14346$.

[35] I-C. Lin, A. P. Seitsonen, I. Tavernelli, M. D. Coutinho-Neto, U. Rothlisberger, 2008, submitted.

[36] A. Tkatchenko, O. A. von Lilienfeld, Phys. Rev. B 2006, 73, 153406.

[37] O. A. von Lilienfeld, D. Andrienko, $J$. Chem. Phys. 2006, 124, 054307.

[38] R. G. Endres, D. L. Cox, R. R. P. Singh, Rev. Mod. Phys 2004, 76, 195.

[39] E. Yavin, E. D. A. Stemp, V. L. O'Shea, S S. David, J. K. Barton, Proc. Natl. Acad. Sci. USA 2006, 103, 3610. 